\title{
On t-Neighbourhoods in Trigonometric Topological Spaces
}

\section{${ }^{1}$ S. Malathi, ${ }^{2}$ Dr. R. Usha Parameswari\& ${ }^{3}$ S. Malathi}

${ }^{1}$ Research Scholar ,(Reg. No: 19222072092001),

${ }^{2}$ Assistant Professor,, ${ }^{1,2}$ Department of Mathematics, Govindammal Aditanar College for Women, Tiruchendur, Affiliated to Manonmaniam Sundaranar University, Abishekapatti, Tirunelveli-627 012, India. ${ }^{1}$ malathis2795@gmail.com , ${ }^{2}$ rushaparameswari@gmail.com ${ }^{3}$ Assistant Professor,Department of Mathematics, Wavoo Wajeeha Women's College of Arts and Science, Kayalpatnam-628 204, India.

Article History:Received:11 January 2021; Accepted: 27 February 2021; Published online: 5 April 2021

Abstract: In this paper we introduce a new type of neighbourhoods, namely, t-neighbourhoods in trigonometric topological spaces and study their basic properties. Also, we discuss the relationship between neighbourhoods and t-neighbourhoods. Further, we give the necessary condition for t-neighbourhoods in trigonometric topological spaces. .

Keywords: t-open; t-closed; t-neighbourhood

\section{Introduction}

In this paper, we introduce t-neighbourhoods in Trigonometric topological spaces. These spaces are based on Sine and Cosine topologies. In a bitopological space we have consider two different topologies but in a trigonometric topological space the two topologies are derived from one topology. From this, we observe that trigonometric topological space is different from bitopological space.

Section 2 deals with the preliminary concepts. In section 3, t-neighbourhoods are introduced and study their basic properties.

\section{Preliminaries}

Throughout this paper $X$ denotes a set having elements from $\left[0, \frac{\pi}{2}\right]$. If $(X, \tau)$ is a topological space, then for any subset $\mathrm{A}$ of $\mathrm{X}, \mathrm{X} \backslash \mathrm{A}$ denotes the complement of $\mathrm{A}$ in $\mathrm{X}$. The following definitions are very useful in the subsequent sections.

Definition: 2.1 [2] Let $X$ be any non-empty set having elements from $\left[0, \frac{\pi}{2}\right]$ and $\tau$ be the topology on $X$. Let SinX be the set consisting of the Sine values of the corresponding elements of $X$. Define a function $f_{s}: X \rightarrow \operatorname{Sin} X$ by $f_{s}(x)=\operatorname{Sin} x$. Then $f_{s}$ is a bijective function. This implies, $f_{s}(\phi)=\phi$ and $f_{s}(X)=\operatorname{Sin} X$. That is, Sin $\phi=\phi$.

Let $\tau_{s}$ be the set consisting of the images (under $f_{s}$ ) of the corresponding elements of $\tau$. Then $\tau_{s}$ form a topology on $\mathrm{f}_{\mathrm{s}}(\mathrm{X})=\operatorname{Sin} \mathrm{X}$. This topology is called a Sine topology (briefly, Sin-topology) of $\mathrm{X}$. The space $\left(\operatorname{Sin} X, \tau_{\mathrm{s}}\right)$ is said to be a Sine topological space corresponding to $\mathrm{X}$.

The elements of $\tau_{\mathrm{s}}$ are called Sin-open sets. The complement of Sin-open sets is said to be Sin-closed. The set of all Sin-closed subsets of $\operatorname{SinX}$ is denoted by $\tau_{s}^{c}$.

Definition: 2.2 [2] Let $X$ be any non-empty set having elements from $\left[0, \frac{\pi}{2}\right]$ and $\tau$ be the topology on $X$. Let $\operatorname{Cos} \mathrm{X}$ be the set consisting of the Cosine values of the corresponding elements of $\mathrm{X}$. Define a function $\mathrm{f}_{\mathrm{c}}: \mathrm{X} \rightarrow \operatorname{Cos} \mathrm{X}$ by $\mathrm{f}_{\mathrm{c}}(\mathrm{x})=\operatorname{Cos} \mathrm{x}$. Then $\mathrm{f}_{\mathrm{c}}$ is bijective. Also, $\mathrm{f}_{\mathrm{c}}(\phi)=\phi$ and $\mathrm{f}_{\mathrm{c}}(\mathrm{X})=\operatorname{Cos} \mathrm{X}$. This implies, $\operatorname{Cos} \phi=\phi$.

Let $\tau_{\mathrm{cs}}$ be the set consisting of the images (under $f_{c}$ ) of the corresponding elements of $\tau$. Then $\tau_{\mathrm{cs}}$ form a topology on CosX. This topology is called Cosine topology (briefly, Cos-topology) of X. The pair (CosX, $\left.\tau_{\mathrm{cs}}\right)$ is called the Cosine topological space corresponding to X. The elements of $\tau_{\mathrm{cs}}$ are called Cos-open sets. The complement of the Cos-open set is said to be Cos-closed. The set of all Cos-closed subsets of Cos X is denoted by $\tau_{c s}^{c}$.

Definition: 2.3 [2] Let $X$ be a non-empty set having elements from $\left[0, \frac{\pi}{2}\right]$. Define $T_{u}(X)$ by $T_{u}(X)=\operatorname{SinX} \cup \operatorname{Cos} X$ and $\mathrm{T}_{\mathrm{i}}(\mathrm{X})$ by $\mathrm{T}_{\mathrm{i}}(\mathrm{X})=\operatorname{Sin} \mathrm{X} \cap \operatorname{Cos} \mathrm{X}$. 
Definition: 2.4 [2] Let $X$ be a non-empty set having elements from $\left[0, \frac{\pi}{2}\right]$ and $\tau$ be the topology on $X$. We define a set $\mathcal{T}=\left\{\phi, U \cup V \cup T_{i}(X): U \in \tau_{s}\right.$ and $\left.V \in \tau_{c s}\right\}$. Then $\mathcal{T}$ form a topology on $T_{u}(X)$. This topology is called trigonometric topology on $\mathrm{T}_{\mathrm{u}}(\mathrm{X})$. The pair $\left(\mathrm{T}_{\mathrm{u}}(\mathrm{X}), \mathcal{T}\right)$ is called a trigonometric topological space. The elements of $\mathcal{T}$ are called trigonometric open sets (briefly, t-open sets). The complement of a trigonometric open set is said to be a trigonometric closed (briefly, t-closed) set. The set of all trigonometric closed sets is denoted by $\mathcal{T}^{\mathrm{c}}$.

\section{3. t-neighbourhoods}

In this section we study about t-neighbourhoods in Trigonometric topological spaces. Throughout this section $\mathrm{T}_{\mathrm{u}}(\mathrm{X})$ denotes the trigonometric topological space with trigonometric topology $\mathcal{T}$.

Definition: 3.1 Let $T_{u}(X)$ be a trigonometric topological space. A subset $N$ of $T_{u}(X)$ is said to be a tneighbourhood (briefly, $t-n b d$ ) of $y \in T_{u}(X)$ if there exists a t-open set $M$ such that $y \in M \subseteq N$.

Definition: 3.2 Let $T_{u}(X)$ be a trigonometric topological space. A subset $N$ of $T_{u}(X)$ is said to be a tneighbourhood (briefly, $t-n b d$ ) of a subset $A$ of $T_{u}(X)$ if there exists a t-open set $M$ such that $A \subseteq M \subseteq N$.

Example: 3.3 Let $X=\left\{\frac{\pi}{6}, \frac{\pi}{4}, \frac{\pi}{2}\right\}$ with topology $\tau=\left\{\phi,\left\{\frac{\pi}{6}\right\},\left\{\frac{\pi}{2}\right\},\left\{\frac{\pi}{6}, \frac{\pi}{2}\right\}, X\right\}$. Then $T_{u}(X)=\left\{\frac{1}{2}, \frac{1}{\sqrt{2}}, \frac{\sqrt{3}}{2}, 1,0\right\}$ and $\mathcal{T}=\left\{\phi, \mathrm{T}_{\mathrm{i}}(\mathrm{X}),\left\{\frac{1}{\sqrt{2}}, \frac{\sqrt{3}}{2}\right\},\left\{0, \frac{1}{\sqrt{2}}\right\},\left\{\frac{1}{2}, \frac{1}{\sqrt{2}}\right\},\left\{1, \frac{1}{\sqrt{2}}\right\}, \operatorname{Cos} \mathrm{X},\left\{\frac{1}{2}, \frac{1}{\sqrt{2}}, \frac{\sqrt{3}}{2}\right\},\left\{\frac{1}{2}, \frac{1}{\sqrt{2}}, 0\right\},\left\{1, \frac{1}{\sqrt{2}}, \frac{\sqrt{3}}{2}\right\}\right.$, $\left.\left\{1, \frac{1}{\sqrt{2}}, 0\right\}, \operatorname{Sin} X,\left\{\frac{1}{2}, \frac{1}{\sqrt{2}}, \frac{\sqrt{3}}{2}, 0\right\},\left\{1, \frac{1}{\sqrt{2}}, \frac{\sqrt{3}}{2}, 0\right\},\left\{1, \frac{1}{\sqrt{2}}, \frac{1}{2}, \frac{\sqrt{3}}{2}\right\},\left\{1, \frac{1}{\sqrt{2}}, \frac{1}{2}, 0\right\}, \mathrm{T}_{\mathrm{u}}(\mathrm{X})\right\}$. Let $\mathrm{N}=\left\{\frac{1}{\sqrt{2}}, \frac{\sqrt{3}}{2}\right\}$. Then $\mathrm{N}$ is a t-nbd of $\frac{\sqrt{3}}{2}$.

Proposition: 3.4 Let $T_{u}(X)$ be a trigonometric topological space. If $N$ is a proper subset of $T_{i}(X)$, then $N$ is not a t-nbd of any point of $T_{u}(X)$.

Proof: Assume that $N$ is a proper subset of $T_{i}(X)$. Suppose that $N$ is a $t-n b d$ of $y \in T_{u}(X)$. Then there exists a $t-$ open set $M$ such that $y \in M \subseteq N$. This implies, $M$ is a proper subset of $T_{i}(X)$. This contradicts the fact that every topen set containing $T_{i}(X)$. Therefore, $N$ is not a t-nbd of any point of $T_{u}(X)$.

Definition: 3.5 Let $\mathrm{T}_{\mathrm{u}}(\mathrm{X})$ be a trigonometric topological space and $\mathrm{N}$ be a subset of $\mathrm{X}$. Define the set $\mathrm{N}^{*}$ by $\mathrm{N}^{*}=\operatorname{SinNUCosN} U \mathrm{~T}_{\mathrm{i}}(\mathrm{X})$. Then $\mathrm{N}^{*}$ is a subset of $\mathrm{T}_{\mathrm{u}}(\mathrm{X})$.

Proposition: 3.6 Let $\mathrm{T}_{\mathrm{u}}(\mathrm{X})$ be a trigonometric topological spaces and $\mathrm{N}, \mathrm{M}$ be a subset of $\mathrm{X}$. Then

1. If $\mathrm{N}$ is open in $\mathrm{X}$, then $\mathrm{N}^{*}$ is t-open in $\mathrm{T}_{\mathrm{u}}(\mathrm{X})$,

2. If $\mathrm{N} \subseteq \mathrm{M}$, then $\mathrm{N}^{*} \subseteq \mathrm{M}^{*}$.

Proof: The proof follows directly from the definition.

Proposition: 3.7 Let $T_{u}(X)$ be a trigonometric topological space. If $N$ is a neighbourhood of $x$, then $N^{*}$ is a t-nbd of Sin $\mathrm{x}$ and $\operatorname{Cos} \mathrm{x}$.

Proof: Assume that $\mathrm{N}$ is a neighbourhood of $\mathrm{x}$. Then there exists an open set $\mathrm{M}$ such that $\mathrm{x} \in \mathrm{M} \subseteq \mathrm{N}$. This implies, Sin $x \in \operatorname{Sin} M \subseteq \operatorname{Sin} N$ and $\operatorname{Cos} x \in \operatorname{Cos} M \subseteq C o s ~ N$. This implies, Sin $x \in \operatorname{Sin} M \cup \operatorname{Cos} M \cup T_{i}(X) \subseteq S i n N U C o s N_{i}(X)$ and $\operatorname{Cos} x \in \operatorname{Sin} M \cup \operatorname{Cos} \mathrm{MUT}_{\mathrm{i}}(\mathrm{X}) \subseteq \operatorname{Sin} \mathrm{N} \cup \operatorname{Cos}_{\mathrm{N}} \cup \mathrm{T}_{\mathrm{i}}(\mathrm{X})$. That is, Sin $\mathrm{x} \in \mathrm{M}^{*} \subseteq \mathrm{N}^{*}$ and $\operatorname{Cos} \mathrm{x} \in \mathrm{M}^{*} \subseteq \mathrm{N}^{*}$. Since $\mathrm{M}$ is open in $\mathrm{X}$, we have $\mathrm{M}^{*}$ is t-open. Therefore, $\mathrm{N}^{*}$ is a t-nbd of $\operatorname{Sin} \mathrm{x}$ and $\operatorname{Cos} \mathrm{X}$.

Proposition: 3.8 Let $T_{u}(X)$ be a trigonometric topological space. If $N$ is a neighbourhood of any point $x \in X$, then $\mathrm{N}^{*}$ is a t-nbd of every point of $\mathrm{T}_{\mathrm{i}}(\mathrm{X})$. 
Proof: Assume that the subset $\mathrm{N}$ of $\mathrm{X}$ is a neighbourhoods of $\mathrm{x}$. Then $\mathrm{N}^{*}$ is a t-nbd of Sin $\mathrm{x}$ and Cos $\mathrm{x}$. Then by Proposition 3.7, $N^{*}$ contains $T_{i}(X)$. Therefore, for each $y \in T_{i}(X)$, we have $y \in T_{i}(X) \subseteq N^{*}$. Hence $N^{*}$ is a $t-n b d$ of every point of $\mathrm{T}_{\mathrm{i}}(\mathrm{X})$.

Proposition: 3.9 Let $\mathrm{T}_{\mathrm{u}}(X)$ be a trigonometric topological space. Then $\mathrm{T}_{\mathrm{i}}(\mathrm{X})$ is a t-nbd of each of its points. Proof: For each point $x \in T_{i}(X)$, there exists a t-open set $T_{i}(X)$ such that $x \in T_{i}(X) \subseteq T_{i}(X)$. Therefore, $T_{i}(X)$ is a tnbd of each of its points.

Proposition: 3.10 Let $\mathrm{T}_{\mathrm{u}}(\mathrm{X})$ be a trigonometric topological space. Then $\mathrm{N}$ is a t-open set if and only if $\mathrm{N}$ is a $\mathrm{t}$ nbd of each of its points.

Proof: Assume that $\mathrm{N}$ is t-open. Let $\mathrm{y} \in \mathrm{N}$. Then $\mathrm{N}$ is a t-open set and $\mathrm{y} \in \mathrm{N} \subseteq \mathrm{N}$. This implies, $\mathrm{N}$ is a $\mathrm{t}-\mathrm{nbd}$ of $\mathrm{y}$. Since $y \in N$ is arbitrary, we have $\mathrm{N}$ is a t-nbd of each of its points. Conversely, assume that $\mathrm{N}$ is a $\mathrm{t}-\mathrm{nbd}$ of each of its points. Then for each point $y_{i}$ of $N$, there exists a t-open set $M_{i}$ such that $y_{i} \in M_{i} \subseteq N$. This implies, $N$ is the union of $\mathrm{M}_{\mathrm{i}}$. Therefore, $\mathrm{N}$ is t-open.

Remark: 3.11 If $\mathrm{N}$ is a $\mathrm{t}$-nbd of some of its points, then $\mathrm{N}$ need not be a t-open set. For example, Consider $\mathrm{X}=\left\{\frac{\pi}{6}, \frac{\pi}{4}, \frac{\pi}{2}\right\} \quad$ with $\tau=\{\phi, X\}$. Then $\mathrm{T}_{\mathrm{u}}(\mathrm{X})=\left\{\frac{1}{2}, \frac{1}{\sqrt{2}}, \frac{\sqrt{3}}{2}, 1,0\right\}$ and $\mathcal{T}=\left\{\phi, \mathrm{T}_{\mathrm{i}}(\mathrm{X}), \operatorname{SinX}, \operatorname{Cos} \mathrm{X}, \mathrm{T}_{\mathrm{u}}(\mathrm{X})\right\}$. Let $\mathrm{N}=\left\{\frac{1}{\sqrt{2}}, 1,0\right\}$ be a subset of $\mathrm{T}_{\mathrm{u}}(\mathrm{X})$. Then $\mathrm{N}$ is a t-nbd of $\frac{1}{\sqrt{2}}$. But it is not a t-open set.

Proposition: 3.12 Let $T_{u}(X)$ be a trigonometric topological space. If $A$ is a t-closed subset of $T_{u}(X)$ and $y \notin A$, then there exists a t-nbd $\mathrm{N}$ of $\mathrm{y}$ such that $\mathrm{N} \cap \mathrm{A}=\phi$.

Proof: Let $\mathrm{A}$ be a t-closed set and $\mathrm{y} \notin \mathrm{A}$. Let $\mathrm{N}=\mathrm{T}_{\mathrm{u}}(\mathrm{X}) \backslash \mathrm{A}$. Then $\mathrm{N}$ is a t-open set containing $\mathrm{y}$. Since every $\mathrm{t}$-open set is a t-nbd of each of its points, we have $\mathrm{N}$ is a t-nbd of $\mathrm{y}$. Also, $\mathrm{N} \cap \mathrm{A}=\phi$.

Definition: 3.13 Let $T_{u}(X)$ be a trigonometric topological space and $y \in T_{u}(X)$. The set of all $t$-nbd of $y$ is called the t-nbd system at $\mathrm{y}$ and is denoted by $\mathrm{t}-\mathrm{N}(\mathrm{y})$.

Proposition: 3.14 Let $\mathrm{T}_{\mathrm{u}}(X)$ be a trigonometric topological space and $y \in \mathrm{T}_{\mathrm{u}}(X)$. Then $\mathrm{t}-\mathrm{N}(\mathrm{y}) \neq \phi$ for every $\mathrm{y} \in \mathrm{T}_{\mathrm{u}}(\mathrm{X})$.

Proof: Since $T_{u}(X)$ is the t-open set, we have $T_{u}(X)$ is the t-nbd of each of its points. Therefore, for every point $y$ of $\mathrm{T}_{\mathrm{u}}(\mathrm{X}), \mathrm{t}-\mathrm{N}(\mathrm{y}) \neq \phi$.

Proposition: 3.15 Let $T_{u}(X)$ be a trigonometric topological space and $y \in T_{u}(X)$. If $N \in t-N(y)$, then $y \in N$.

Proof: If $\mathrm{N} \in \mathrm{t}-\mathrm{N}(\mathrm{y})$, then $\mathrm{N}$ is a $\mathrm{t}-\mathrm{nbd}$ of $\mathrm{y}$. This implies, $\mathrm{y} \in \mathrm{N}$.

Proposition: 3.16 Let $T_{u}(X)$ be a trigonometric topological space and $y \in T_{u}(X)$. If $N \in t-N(y)$ and $N \subseteq M$, then $\mathrm{M} \in \mathrm{t}-\mathrm{N}(\mathrm{y})$.

Proof: Assume that $\mathrm{N} \in \mathrm{t}-\mathrm{N}(\mathrm{y})$ and $\mathrm{N} \subseteq \mathrm{M}$. Then $\mathrm{N}$ is a $\mathrm{t}$-nbd of $\mathrm{y}$. Therefore, there exists a t-open set $\mathrm{W}$ such that $\mathrm{y} \in \mathrm{W} \subseteq \mathrm{M}$. This implies, $\mathrm{M}$ is a $\mathrm{t}-\mathrm{nbd}$ of $\mathrm{y}$. Hence $\mathrm{M} \in \mathrm{t}-\mathrm{N}(\mathrm{y})$.

Proposition: 3.17 Let $T_{u}(X)$ be a trigonometric topological space and $y \in T_{u}(X)$. If $N \in t-N(y)$ and $M \in t-N(y)$, then

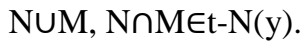

Proof: Assume that $\mathrm{N} \in \mathrm{t}-\mathrm{N}(\mathrm{y})$ and $\mathrm{M} \in \mathrm{t}-\mathrm{N}(\mathrm{y})$. Then there exist t-open sets $\mathrm{A}$ and $\mathrm{B}$ such that $\mathrm{y} \in \mathrm{A} \subseteq \mathrm{N}$ and $\mathrm{y} \in \mathrm{B} \subseteq \mathrm{M}$. This implies, $\mathrm{y} \in \mathrm{A} \cap \mathrm{B} \subseteq \mathrm{N} \cap \mathrm{M}$ and $\mathrm{y} \in \mathrm{A} \cup \mathrm{B} \subseteq N \cup M$. Since $A$ and $\mathrm{B}$ are $\mathrm{t}$-open, we have $\mathrm{A} \cap \mathrm{B}$ and $A \cup B$

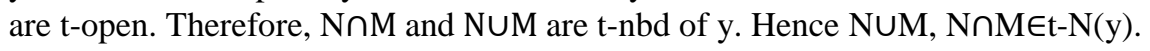


Proposition: 3.18 Let $T_{u}(X)$ be a trigonometric topological space and $y \in T_{u}(X)$. If $N \in t-N(y)$, then there exists $\mathrm{M} \in \mathrm{t}-\mathrm{N}(\mathrm{y})$ such that $\mathrm{M} \subseteq \mathrm{N}$ and $\mathrm{M} \in \mathrm{t}-\mathrm{N}(\mathrm{x})$ for every $\mathrm{x} \in \mathrm{M}$.

Proof: Assume that $\mathrm{N} \in \mathrm{t}-\mathrm{N}(\mathrm{y})$. Then there exists a t-open set $\mathrm{M}$ such that $\mathrm{y} \in \mathrm{M} \subseteq \mathrm{N}$. Since $\mathrm{M}$ is t-open, we have $M$ is a $t-n b d$ of each of its points. Therefore, $M \in t-N(y)$ and $M \in t-N(x)$ for every $x \in M$.

\section{Conclusion:}

In this paper we have introduced t-neighbourhoods in Trigonometric Topological Spaces and studied some of their basic properties.

\section{References}

1. James R. Munkres (2002), "Topology (Second edition)”, Prentice-Hall of India Private Limited, New Delhi.

2. S. Malathi and R. Usha Parameswari, "On Trigonometric Topological spaces", Advances in Mathematics: Scientific Journal, Vol. 9, No. 5, (2020), 2477-2488.

3. G.F. Simmons (1968), "Introduction to Topology and Modern Analysis", McGraw-Hill Book Company, New York. 